\title{
Two Spatially Distinct Posterior Alpha Sources Fulfill Different Functional Roles in Attention
}

\author{
ㅈodika Sokoliuk, ${ }^{1}$ Stephen D. Mayhew, ${ }^{1}$-Kevin M. Aquino, ${ }^{2,3}$-Ross Wilson, ${ }^{1}$ Matthew J. Brookes, ${ }^{2}$ \\ [DSusan T. Francis, ${ }^{2}$ SSimon Hanslmayr, ${ }^{1}$ and ${ }^{-}$Karen J. Mullinger ${ }^{1,2}$ \\ ${ }^{1}$ Centre for Human Brain Health, School of Psychology, University of Birmingham, B15 2TT, Edgbaston, Birmingham, United Kingdom, ${ }^{2}$ Sir Peter \\ Mansfield Imaging Centre, School of Physics and Astronomy, University of Nottingham, NG7 2QX, Nottingham, United Kingdom, and ${ }^{3}$ Monash University, \\ 3800 Melbourne, Australia
}

Directing attention helps to extract relevant information and suppress distracters. Alpha brain oscillations $(8-12 \mathrm{~Hz})$ are crucial for this process, with power decreases facilitating processing of important information and power increases inhibiting brain regions processing irrelevant information. Evidence for this phenomenon arises from visual attention studies (Worden et al., 2000); however, the effect also exists in other modalities, including the somatosensory system (Haegens et al., 2011) and intersensory attention tasks (Foxe and Snyder, 2011). We investigated in human participants ( 10 females, 10 males) the role of alpha oscillations in focused (0/100\%) versus divided $(40 / 60 \%)$ attention, both across modalities (visual/somatosensory; Experiment 1) and within the same modality (visual domain: across hemifields; Experiment 2) while recording EEG over 128 scalp electrodes. In Experiment 1, participants divided their attention between visual and somatosensory modality to determine the temporal/spatial frequency of a target stimulus (vibrotactile stimulus/Gabor grating). In Experiment 2, participants divided attention between two visual hemifields to identify the orientation of a Gabor grating. In both experiments, prestimulus alpha power in visual areas decreased linearly with increasing attention to visual stimuli. In contrast, prestimulus alpha power in parietal areas was lower when attention was divided between modalities/hemifields compared with focused attention. These results suggest there are two alpha sources, one of which reflects the "visual spotlight of attention" and the other reflects attentional effort. To our knowledge, this is the first study to show that attention recruits two spatially distinct alpha sources in occipital and parietal brain regions, acting simultaneously but serving different functions in attention.

Key words: alpha oscillations; attention; brain oscillations; EEG; multimodal attention; source estimates

Significance Statement

Attention to one spatial location/sensory modality leads to power changes of alpha oscillations $(\sim 10 \mathrm{~Hz})$ with decreased power over regions processing relevant information and power increases to actively inhibit areas processing "to-be-ignored" information. Here, we used detailed source modeling to investigate EEG data recorded during separate unimodal (visual) and multimodal (visual and somatosensory) attention tasks. Participants either focused their attention on one modality/spatial location or directed it to both. We show for the first time two distinct alpha sources are active simultaneously but play different roles. A sensory (visual) alpha source was linearly modulated by attention representing the "visual spotlight of attention." By contrast, a parietal alpha source was modulated by attentional effort, showing lowest alpha power when attention was divided.

\section{Introduction}

Allocation of attention helps to extract important and neglect irrelevant information. Alpha brain oscillations $(8-13 \mathrm{~Hz})$

This work was supported by the Leverhulme Trust (Research Project Grant to K.J.M. RPG-2014-369).

The authors declare no competing financial interests.

Correspondence should be addressed to Rodika Sokoliuk at r.sokoliuk@bham.ac.uk. potentially occupy this filtering role and lead to excitation or inhibition of sensory-specific regions, thereby facilitating or suppressing sensory processing (Klimesch et al., 2007; Jensen and Mazaheri, 2010; Mathewson et al., 2011). When attending to two spatial locations (right/left), a relative alpha power decrease is observed over brain regions processing relevant information compared with regions inhibiting irrelevant information. Such a hemispheric alpha power lateralization over occipitoparietal re- 
gions has been shown many times in visuospatial attention (Foxe et al., 1998; Worden et al., 2000; Kelly et al., 2006; Thut et al., 2006; Gould et al., 2011; Zumer et al., 2014). This has also been observed in the somatosensory system (Anderson and Ding, 2011; Haegens et al., 2011, 2012; van Ede et al., 2011) and in intersensory attention (Foxe and Snyder, 2011; Gomez-Ramirez et al., 2011; Bauer et al., 2012).

What happens if attention is divided between two sensory modalities simultaneously? Would this provoke an alpha-power imbalance between sensory-specific regions reflecting the peak location of attention like that recently observed for spatially divided visual attention (Gould et al., 2011)? Existing literature showed evidence for alpha-power modulation over sensoryspecific brain regions; however, attention was not divided between two senses simultaneously (Foxe and Snyder, 2011; Gomez-Ramirez et al., 2011; Bauer et al., 2012). Functional magnetic resonance imaging ( $\mathrm{fMRI}$ ) evidence suggests attention also modulates activity over higher-level frontal and parietal areas (Corbetta and Shulman, 2002) that modulate lower-level sensory regions via top-down control (Bressler et al., 2008). Inhibiting frontal eye field (FEF) and inferior parietal lobule (IPL) using repetitive transcranial magnetic stimulation (rTMS), Capotosto et al. (2009) observed increased reaction times (RTs) and decreased accuracy for visual detection and thereby confirmed fMRI results. They concluded that inhibiting these regions disrupted the control over visual alpha oscillations and altered behavior. According to the authors, both primary sensory and parietal regions are important for controlling attention allocation. Hints of this in EEG are shown by the spatial and functional dissociation of occipital and parietal alpha sources during visual perception (Gulbinaite et al., 2017).

Here, we investigated potential differences in the role of alpha oscillations in focused (0/100\%) versus divided (40/60\%) attention, both across modalities (visual/somatosensory) and within a modality (visual: across hemifields). We used multimodal (visual/somatosensory, Experiment 1) and unimodal (left/right visual fields, Experiment 2) attention paradigms while recording scalp EEG over 128 electrodes.

A linearly constrained minimum variance (LCMV) beam former (Van Drongelen et al., 1996) source localized changes in prestimulus alpha power. Two alpha sources were identified in Experiment 1: A visual source decreased linearly in power with increasing attention to visual stimuli; a second source in the parietal cortex modulated by task difficulty showed lower alpha power when attention was divided between modalities. Experiment 2 shared the visual source with linear attention modulation however parietal brain regions were not as strongly modulated.

To our knowledge, this study is the first to reveal two spatially distinct alpha mechanisms acting simultaneously and yet performing different roles in attention: a sensory, visual alpha source reflecting the current location of attention and a parietal alpha source modulated by task difficulty and reflecting attentional effort.

\section{Materials and Methods \\ Participants}

Data were acquired from 20 healthy participants (all right-handed, 10 females, mean age $28.1 \pm 3.8$ years) with normal or corrected to normal vision. One participant was not included in final data analysis because of the absence of an anatomical MRI scan that prohibited complete data analysis. Fifteen of these 20 participants performed two attention paradigms (Experiments 1 and 2) and the remaining four subjects only participated in Experiment 1. Therefore, Experiment 1 had 19 subjects in total and Experiment 2 had 15 in total.
The study was approved by the University of Birmingham Research Ethics Committee. Before the start of the experiment(s), participants provided informed written consent.

\section{Stimuli and task}

Visual and somatosensory stimuli were presented using Psychophysics Toolbox (version 3; Brainard, 1997) running in MATLAB (version 2014b; The MathWorks) on a desktop computer (Windows 7 operating system). Participants sat comfortably in a dark room. To minimize head movement and to maintain a constant degree of visual angle for the visual stimuli, their heads were kept stable using a chin rest.

Visual stimuli were presented in Experiments 1 and 2 on a gray background. Gabor gratings were presented briefly (presentation time: 66.7 ms; radius: 1.75 degrees of visual angle, phase: $\left.180^{\circ}\right)$, on a gray background at a distance of $57 \mathrm{~cm}$, using a cathode ray monitor (resolution: $600 \times 800$ pixels). These stimuli were presented vertically centered and with a horizontal eccentricity of \pm 8 degrees of visual angle from a horizontally centered white fixation point (radius: 0.1 degrees of visual angle). In Experiment 2, two Gabor gratings were presented to the left and right of the fixation point whereas in Experiment 1 a single visual stimulus was presented to the left of the fixation point, simultaneously with a 250-ms-long vibrotactile stimulus to the tip of the left index finger using a piezoelectric stimulator (Dancer Design).

In Experiment 1, the multimodal attention task was conducted (Fig. $1 A)$. Although fixating on the fixation cross, subjects had to covertly divide their attention between two sensory modalities, attending either more to visual or more to somatosensory stimuli $(0 / 100 \%$ or $40 / 60 \%$ attention toward somatosensory/visual domain and vice-versa). A visual cue $(5 \times 2.5$ degrees of visual angle) was presented at fixation at the beginning of every trial for $250 \mathrm{~ms}$, indicating how attention was to be divided. Cues took the form of black arrows indicating the likelihood of subsequent target appearance in each modality (cf. Fig. 1A). After an asynchronous interstimulus interval (aISI) of 1.3-1.6 s (aISIs were randomly chosen for every trial reaching from $1.3 \mathrm{~s}$ (minimum aISI) to $1.6 \mathrm{~s}$ (maximum aISI)), during which participants were required to divide their attention between modalities according to the prestimulus cue, visual and somatosensory stimuli were presented simultaneously. Gabor patterns were presented in a tilted orientation: for half of the participants they were tilted at $45^{\circ}$ and for the other half at $-45^{\circ}$. Stimuli with a low or high spatial frequency $(0.025$ cycles/pixel and 0.1 cycles/pixel $)$ were visual targets and medium-frequency stimuli $(0.05$ cycles/pixel $)$ were visual distracters. In the somatosensory domain, vibrotactile stimulation at a low or high temporal frequency ( 4 and $52 \mathrm{~Hz}$ ) served as somatosensory targets and those at medium temporal frequency $(16 \mathrm{~Hz})$ as somatosensory distracters. In every trial, one target (e.g., a visual Gabor pattern with a high spatial frequency) and one distracter (e.g., a somatosensory stimulus with a medium temporal frequency) stimulus were presented simultaneously. After stimulus presentation, white question marks $(5 \times 1.5$ degrees of visual angle) indicated an $850 \mathrm{~ms}$ response period where participants pressed a button with their right index finger to report the frequency of the target (two different keys: high or low frequency, regardless of probed modality) as quickly as possible. Even if participants were responding before the end of the response period, the next trial only started after $850 \mathrm{~ms}$ with an aISI.

In Experiment 2 the unimodal attention task was conducted (Fig. 1B). This second experiment had a similar structure to Experiment 1 but used only visual Gabor gratings (spatial frequency: 0.05 cycles/pixel), akin to a classic Posner task (Posner et al., 1980). Subjects had to covertly direct their attention in a graded fashion either more to the left or more to the right visual hemifield $(0 / 100 \%, 20 / 80 \%$, or $40 / 60 \%$, attention toward left/right visual hemifields and vice-versa) while they fixated on a central fixation cross (similar to Gould et al., 2011). Trials started with the presentation of a visual cue $(5 \times 2.5$ degrees of visual angle; presentation time: $250 \mathrm{~ms}$ ) in the form of black arrows indicating where subjects should direct their spatial attention (cf. Fig. 1B). As in Experiment 1, this was followed by an aISI of 1.3-1.6 s, before visual stimuli were presented to the left and right of the fixation point. For half of the participants, horizontal and vertical gratings were target stimuli and rightwards $\left(45^{\circ}\right)$ and leftwards $\left(-45^{\circ}\right)$ tilted gratings served as distracters, whereas for the 


\section{A Experiment 1 (multi-modal)}
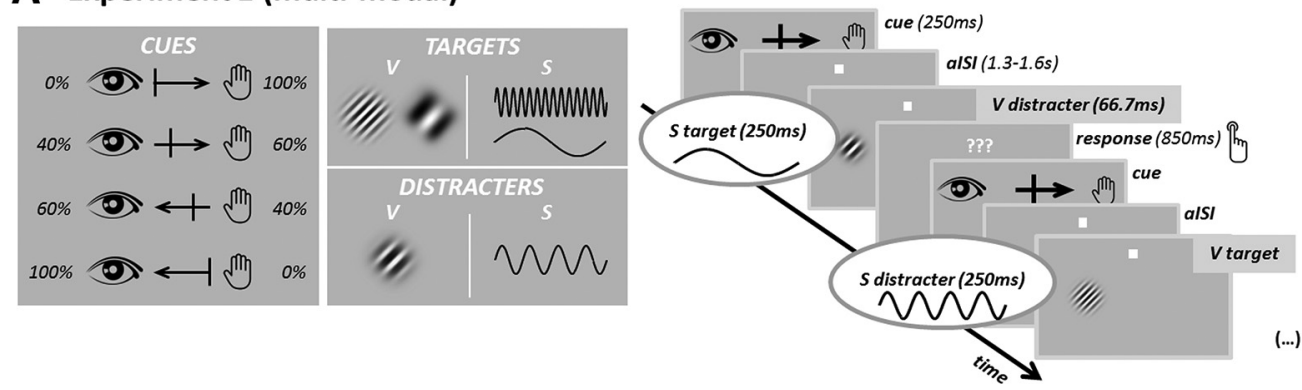

\section{B Experiment 2 (uni-modal)}
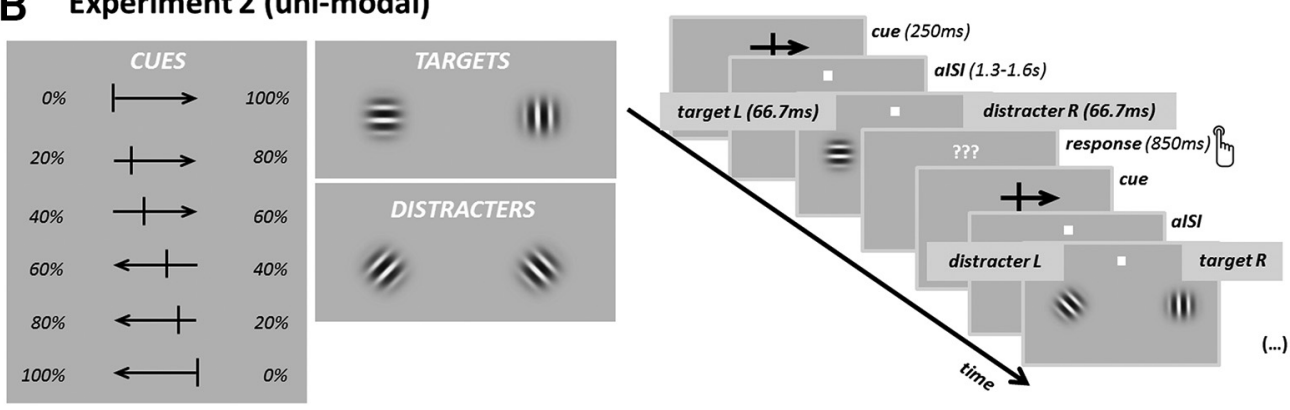

Figure 1. Paradigms of Experiments 1 and 2. A, Attention paradigm used in Experiment 1. Left, Attentional cues used to manipulate participants' attention for the four different attention conditions. The eye represents "attention to the visual system" and the hand represents "attention to the somatosensory system." The arrows point in the direction of the modality that should be more strongly attended to. The numbers (e.g., $0 \%$ ) were not presented during the experiment but are shown here for clarity. Target stimuli in the visual domain were high and low-frequency Gabor patterns, whereas stimuli with a medium spatial frequency represented visual distracters (middle). In the somatosensory domain, stimuli showing a high or a low temporal frequency served as targets whereas medium frequency stimuli were distracters (middle). Right, Temporal sequence of the experiment. A cue was presented for $250 \mathrm{~ms}$ before a blank screen only showing the fixation point for 1.3-1.6 (alSI). Then, both visual $(66.7 \mathrm{~ms})$ and somatosensory stimuli $(250 \mathrm{~ms})$ were presented simultaneously, although only one of them represented the target stimulus. Subjects then had $850 \mathrm{~ms}$ to respond whether the target was high or low frequency before the next trial. $\boldsymbol{B}$, Left, Visual cues used to manipulate participants' attention in the six attention conditions of Experiment 2. The arrows are pointing toward the side of the visual field to which more attention should be paid with dividing lines indicating how attention should be divided (as in Experiment 1). Again numbers (e.g., $0 \%$ ) are only shown for clarity and were not presented. As in Experiment 1, each trial started with the presentation of a visual cue ( $250 \mathrm{~ms}$ ) before a blank screen with only the fixation point was presented for 1.3-1.6 (alSI) (right). Then, stimuli appeared on both sides of the visual field whereat only one of them was a target whose orientation (e.g., "horizontal or vertical", see middle panel) had to be reported within $850 \mathrm{~ms}$ before the next trial started. The middle panel showing target and distractors is an example which was used for half the subjects; for the other half the subjects the target and distractors were the opposite. Note: to facilitate visibility in these schematics, the visual stimuli are larger than the actual size these stimuli occupied on the screen in the experiment.

other half, the opposite was true. In every trial, one target (e.g., horizontal grating) and one distractor (e.g., rightwards tilted grating) appeared simultaneously at opposite sides of the fixation cross. After stimulus presentation, a white question mark was presented for $850 \mathrm{~ms}$ to indicate the response period. The task was to respond as quickly as possible to indicate the orientation of the target grating (two different keys: e.g., horizontal or vertical). Even if participants were responding before the end of the response period, the next trial only started after $850 \mathrm{~ms}$ with an aISI.

In both experiments, participants were given feedback on their performance (accuracy and RT), which was displayed after each experimental run to maintain their motivation for performing the tasks.

Participants completed a training run consisting of 10 trials per attention condition (resulting in a total of 40/60 trials for Experiments 1 and 2, respectively) before they performed the same task in a staircase experimental run, where the contrast of the visual stimuli was adapted according to participants' performance (60 trials per attention condition) to ensure an accuracy of $\sim 80 \%$ was achieved. For somatosensory stimuli, a similar procedure was used to adapt the amplitude of vibrotactile pulses.

The subjects then started the experiment and performed 150 trials per attention condition giving a total of 600/900 trials for Experiments 1 and 2, respectively. Experiments were divided into three individual runs; all runs contained equal number of trials of each attention condition (50 trials/condition/run). All trials of a given attention condition within a run were grouped together in one block, the order of the blocks between runs was varied pseudorandomly. The whole study took $\sim 1.5 \mathrm{~h}$ per participant, including short breaks that the participants took between runs.

\section{EEG data acquisition}

EEG data were recorded from 128 active scalp electrodes following an equi-radial montage at $1024 \mathrm{~Hz}$ sampling rate using a Biosemi EEG system with a reference electrode (common mode sense electrode) placed parietocentrally for the recording. In addition, EOG was recorded using three active ocular electrodes with the horizontal electrodes being placed near the two temples and the vertical electrode below the left eye. In Experiment 1, data were recorded in 3 runs of $\sim 9$ min each; in Experiment 2 , the 3 runs consisted of $\sim 12$ min each.

After each EEG recording session, the individual electrode positions were digitized relative to the surface of the head with a Polhemus FASTRAK using Brainstorm software (Tadel et al., 2011) running in MATLAB. In addition, each subject attended a separate MRI session where a T1-weighted anatomical image (MPRAGE sequence) of the head, including the nose, with $1 \mathrm{~mm}$ isotropic resolution was acquired on either a $3 \mathrm{~T}$ or $7 \mathrm{~T}$ MRI system that was registered with the digitized head shape.

\section{Data analysis}

\section{Behavioral}

Behavioral parameters analyzed were RT and accuracy. To analyze significant differences between attention conditions, a repeated-measures two-way ANOVA was computed for both behavioral parameters and experiments separately, with the following factors: (1) attention condition $(60 \%$ and $100 \%$ for Experiment 1 and $60 \%, 80 \%$, and $100 \%$ for Experiment 2, and (2) attended modality (somatosensory and visual) or hemifield (left and right). Post hoc paired-sample $t$ tests were used to identify individual differences between attention conditions and 
$p$-values were subsequently Bonferroni corrected to account for multiple comparisons.

\section{EEG}

All EEG data processing was performed using the MATLAB toolbox Fieldtrip (Oostenveld et al., 2011).

Preprocessing. Data were read in as continuous data. For each channel, data were notch filtered $(49-51 \mathrm{~Hz})$ to reduce line noise, detrended to remove linear drifts, and demeaned (subtracting the average signal recorded over the whole time course at each channel) to remove between run baseline effects. By visual inspection, noisy channels (i.e., channels with obvious artifacts) were removed from further data analysis. This resulted in a group mean of $( \pm$ SE) $117 \pm 4 / 116 \pm 5$ channels remaining for further analysis for Experiments 1 and 2, respectively. Independent component analysis (ICA, logistic infomax ICA algorithm; cf. Bell and Sejnowski, 1995) was then performed to discard eye blinks from the recorded data, with an average of $1 \pm 0.6$ ICs for Experiment 1 and $1.5 \pm$ 1.5 ICs for Experiment 2 removed from each dataset. The remaining ICs were reprojected to the channel level. Finally, data were rereferenced to the average of all the non-noisy channels that remained for each subject and run.

These data were subsequently used for time-frequency analysis on the sensor and source level.

Sensor-level analysis. Data were epoched into $1.7 \mathrm{~s}(-1.5 \mathrm{~s}$ until $+0.2 \mathrm{~s}$ relative to the stimulus presentation onset) segments for every trial and the separate runs of the experiment concatenated. All trial level data were visually inspected and noisy trials removed for each subject, resulting in $818 \pm 12.4 / 539 \pm 11.7$ (number of trials \pm SEM) trials of data remaining for Experiments 1 and 2. Furthermore, those trials where the subject had responded incorrectly to the target were subsequently removed such that $727 \pm 16.5 / 471 \pm 15.6$ trials remained for Experiments 1 and 2 .

Source level analysis. Individual, four-layer (scalp, skull, CSF, and brain) boundary element (BEM) head models were constructed from the individual subject $\mathrm{T}_{1}$-weighted anatomical images using the Fieldtrip toolbox with the 'dipoli' method (http://www.ru.nl/neuroimaging/fieldtrip) (Oostenveld et al., 2011). Individual electrode positions were aligned to the scalp surface of the subject's $T_{1}$ using the fiducial points and head shape to inform alignment. In four of the 19 participants, no individual electrode positions were recorded due to technical problems; therefore, in these subjects, the average electrode positions of the 11 other participants sharing the same electrode layout were used and warped to the scalp surface extracted from the segmented individual $\mathrm{T}_{1}$-weighted scans.

Beamforming analysis was performed using an LCMV beam former (Van Drongelen et al., 1996; Van Veen et al., 1997; Robinson and Vrba, 1998) implemented in the Fieldtrip toolbox to spatially localize changes in alpha power between different attention conditions. The continuous data for each run were first filtered into the alpha-frequency band (8-13 $\mathrm{Hz}$ ), applying the default parameters for a FIR band-pass filter (which uses the MATLAB firl function with a two-pass filter direction, a hamming filter window type, and a filter order of 768 for 10 subjects [sampling rate: $2048 \mathrm{~Hz}$ ) and 384 (sampling rate: $1024 \mathrm{~Hz}$ ) for the remaining nine subjects]. The filtered data were subsequently investigated for temporal leakage of the peak of the ERP into the prestimulus period, with no leakage found. The data were then epoched $-1.5 \mathrm{~s}$ to $+0.2 \mathrm{~s}$ relative to stimulus onset. The noisy and incorrect response trials, identified from the broadband visual data inspection (see "Sensor level" section above) were removed. Remaining trials were then concatenated over runs, down-sampled to $500 \mathrm{~Hz}$ and beam former weights (also known as a spatial filter) (Van Veen et al., 1997) derived. All attention conditions within an experiment were considered together to calculate these weights as the spatial sources of the alpha power were not hypothesized to change between conditions but only their relative amplitude.

For each subject, the preprocessed, cleaned, and down-sampled sensor level data were then separated into trials for each of the attention conditions. The number of trials in each condition was reduced to match that of the condition with the minimum number of trials remaining. This data rejection process was done by randomly removing trials from conditions containing more trials than the minimum. This process ensured that all source localization comparisons were performed on equal amounts of data to avoid biases. An average of $105 \pm 22$ of the 150 trials per condition for Experiment 1 and $107 \pm 16$ of the 150 trials per conditions for Experiment 2 remained (mean \pm SE over subjects) for further source analysis.

To enable alpha power to be calculated only during the aISI, trials were then segmented, resulting in a time window from $-1.3 \mathrm{~s}$ to $0 \mathrm{~s}$ relative to stimulus onset and concatenated together for each condition to ensure no baseline effects within trials were removed. The source power at each location in the brain BEM $(0.5 \mathrm{~cm}$ grid $)$ was estimated for each condition using the previously derived weights from all conditions. These source power maps were then used to calculate the alpha modulation index (AMI) source maps for both experiments for each subject using Equation 1 , where the source power estimates at each location in the brain for each condition were input, as described previously (Zumer et al., 2014):

$$
A M I=\frac{[\operatorname{SPow}(\text { cond } 1)-\operatorname{SPow}(\text { cond } 2)]}{\{0.5 \times[\operatorname{SPow}(\text { cond } 1)+\operatorname{SPow}(\text { cond } 2)]\}}
$$

In Experiment 1, the AMI between trials where participants focused on one modality compared with focusing on the other, for example, between $100 \%$ attention to the visual domain vs $100 \%$ to the somatosensory domain, was calculated using Equation 1, where SPow ("source power") was calculated for every location in the brain (on the $0.5 \mathrm{~cm}$ grid) and is the power estimate of the alpha band signal over the time period -1.3 to $0 \mathrm{~s}$ relative to stimulus onset for all trials in a given condition. Here, cond 1 denotes attend $100 \%$ to visual (and $0 \%$ to somatosensory) stimuli and cond 2 denotes attend $100 \%$ to somatosensory (and $0 \%$ to visual) stimuli.

Furthermore, the AMI between trials where participants focused on one modality (100\% visual or somatosensory; cond 1 in Eq. 1$)$ and those where attention was divided between modalities (60\% visual (i.e., $60 \%$ visual and $40 \%$ somatosensory) or somatosensory (i.e., $60 \%$ somatosensory and $40 \%$ visual); cond 2 in Eq. 1) was computed. The equivalent AMIs were calculated for Experiment 2. First, attention conditions 100\% left (cond 1 in Eq. 1) and 100\% right (cond 2 in Eq. 1) were compared. Then, trials were compared according to whether subjects paid attention to only one side of the visual field (100\%; cond 1 in Eq. 1) or divided their attention between left and right hemifields (60\%; cond 2 in Eq. 1).

The AMI $(100 \%, 100 \%)$ contrasts " $100 \%$ visual (V) vs $100 \%$ somatosensory (S)" and "100\% left (L) versus 100\% right (R)" for Experiments 1 and 2, respectively, were designed to investigate differences in alpha modulation depending on the attentional cue. The AMI $(100 \%, 60 \%)$ contrasts " $100 \%$ (visual/somatosensory) versus $60 \%$ (visual/somatosensory)" and " $100 \%$ (left/right) versus $60 \%$ (left/right)" for Experiments 1 and 2, respectively, were designed to elucidate whether task difficulty was reflected by modulations in alpha power.

AMI source maps for each subject were spatially normalized to the MNI template before being averaged over subjects for each experiment to provide a grand average. The different grand average AMI source maps were visually inspected for local minima and maxima for the two experiments. In both experiments, local minima and maxima were observed over the visual cortex $[\mathrm{AMI}(100 \%, 100 \%)]$ and the parietal cortex [AMI $(100 \%, 60 \%)]$, respectively. For Experiment 1, all stimuli were presented on the left thus hypothesized to recruit the right hemisphere of the brain primarily. Therefore, the maximum AMI value peak location in the right parietal cortex (anatomically defined) from the AMI(100\%,60\%) maps and a minimum AMI value peak location in the right visual cortex from the $\operatorname{AMI}(100 \%, 100 \%)$ were found for each subject individually. For Experiment 2 bilateral stimulus presentation resulted in hypothesized responses in both hemispheres. Therefore, the AMI maxima were identified in the right and left parietal cortices [ $\operatorname{AMI}(100 \%, 60 \%)]$, and in the left visual cortex $[\mathrm{AMI}(100 \%, 100 \%)]$. Furthermore, the AMI minimum in the right visual cortex was identified $[\operatorname{AMI}(100 \%, 100 \%)]$. All peak locations within the anatomically defined regions were identified for each subject individually.

Peak location analysis. The identified peak locations were used as virtual electrode (VE) locations from which alpha frequency time courses were extracted for each participant individually. Time courses were ob- 
Table 1. AAL regions with MNI coordinates of center of mass investigated in Experiment 1

\begin{tabular}{|c|c|c|c|}
\hline \multirow[b]{2}{*}{ AAL region in right hemisphere (Exp. 1) } & \multicolumn{3}{|c|}{ Centre of mass MNI coordinates $[\mathrm{mm}](x / y / z)$} \\
\hline & $x$ & $y$ & $Z$ \\
\hline Precentral gyrus & 35 & -10 & 50 \\
\hline Angular gyrus & 40 & 60 & 35 \\
\hline Calcarine gyrus & 10 & -75 & 5 \\
\hline Cuneus & 5 & -80 & 25 \\
\hline Fusiform gyrus & 30 & -45 & -20 \\
\hline Inferior occipital gyrus & 35 & -75 & -10 \\
\hline Inferior parietal lobule & 40 & -45 & 45 \\
\hline Lingual gyrus & 15 & -65 & -5 \\
\hline Medial occipital gyrus & 30 & -75 & 15 \\
\hline Parietocentral lobule & 5 & -35 & 65 \\
\hline Precuneus & 10 & -55 & 40 \\
\hline Postcentral gyrus & 35 & -30 & 50 \\
\hline Superior medial gyrus & 55 & -35 & 30 \\
\hline Superior occipital gyrus & 20 & -80 & 25 \\
\hline Superior parietal gyrus & 25 & -60 & 55 \\
\hline
\end{tabular}

tained at each VE location by multiplying the cleaned, continuous, down-sampled channel level data (used to derive the initial weights) by the respective alpha beam former weights derived over all data (see above). Time courses were then demeaned before a Hilbert transform was performed to provide a measure of alpha power at each VE location interrogated for each subject. The data were then epoched $-1.3 \mathrm{~s}$ to $0 \mathrm{~s}$ relative to stimulus onset (i.e., the aISI period) and separated into conditions (using the same balancing procedure used to derive the source maps). The average alpha power over trials for each condition was found and then averaged over the aISI period $(-1.3-0 \mathrm{~s})$ to provide a measure of mean alpha power per condition in the visual and parietal cortices.

For Experiment 2, data from left and right hemispheres were combined by flipping the attention conditions (attention left 100\% = attention right $100 \%$ etc.) for the data recorded over the right hemisphere, effectively resulting in alpha power modulations from the left parietal and visual cortices (cf. Waldhauser et al., 2016). This procedure was designed to increase the signal-to-noise ratio.

To take into account between-subject variance, alpha power values were then normalized by the maximum average alpha power value in any condition for each subject. Subsequently, the grand average over subjects was computed. These were tested for linear and quadratic modulation over conditions by fitting the data first to linear and then to quadratic functions using the MATLAB function polyfitn.

Automated anatomical labeling ( $A A L)$ analysis. To test whether the linear and quadratic modulations observed from the peak location analysis were statistically significant, we performed additional analyses based purely on anatomically parcellated brain regions and therefore not biased by the AMI source maps in identification of locations to interrogate. Brain regions were parcellated using the AAL atlas (Tzourio-Mazoyer et al., 2002). Only the anatomical regions in which an alpha related response was predicted were interrogated. Therefore $15 \mathrm{AAL}$ regions in the right hemisphere, spanning from the visual cortex to the somatosensory cortex and $26 \mathrm{AAL}$ regions in right and left hemisphere, reaching from visual to parietal cortex, were investigated in Experiments 1 and 2, respectively (Tables 1, 2).

The following analysis approach, previously used on MEG data (Brookes et al., 2016), was used here. For each subject, all AAL regions were warped onto the individual subject's $\mathrm{T}_{1}$-weighted image and time courses were then extracted from all VE locations (on a $0.5 \mathrm{~cm}$ grid) which fell within the AAL regions. The VE time courses were extracted using the same data and processes used for the peak location analysis. Time courses from VE locations (each grid point) were weighted according to the Euclidian distance of the VE location to the center of gravity of the respective AAL region. After applying the correct weighting, time course data were summed over all VEs per AAL region, to give one time course per AAL region containing all trials, which was then demeaned. The Hilbert transform was subsequently applied to time courses for each
Table 2. AAL regions with MNI coordinates of center of mass investigated in Experiment 2

\begin{tabular}{lccc}
\hline \multirow{2}{*}{$\begin{array}{l}\text { AAL region in right and left hemisphere } \\
\text { (Exp. 2) }\end{array}$} & \multicolumn{3}{c}{ Centre of mass MNI coordinates $(\mathrm{mm})(x / \mathrm{y} / \mathrm{z})$} \\
\cline { 2 - 4 } R/L angular gyrus & $40 /-40$ & $60 /-60$ & $35 / 35$ \\
R/L calcarine gyrus & $10 /-15$ & $-75 /-75$ & $5 / 10$ \\
R/L cuneus & $5 /-15$ & $-80 /-80$ & $25 / 25$ \\
R/L fusiform gyrus & $30 /-35$ & $-45 /-45$ & $-20 /-20$ \\
R/L inferior occipital gyrus & $35 /-35$ & $-75 /-80$ & $-10 /-10$ \\
R/L inferior parietal lobule & $40 /-40$ & $-45 /-45$ & $45 / 50$ \\
R/L lingual gyrus & $15 /-15$ & $-65 /-65$ & $-5 /-5$ \\
R/L medial occipital gyrus & $30 /-35$ & $-75 /-75$ & $15 / 20$ \\
R/L postcentral lobule & $10 /-5$ & $-25 /-35$ & $65 / 65$ \\
R/L precuneus & $10 /-10$ & $-55 /-55$ & $40 / 40$ \\
R/L superior medial gyrus & $55 /-50$ & $-35 /-35$ & $30 / 35$ \\
R/L superior occipital gyrus & $20 /-25$ & $-80 /-75$ & $25 / 30$ \\
R/L superior parietal gyrus & $25 /-25$ & $-60 /-55$ & $55 / 55$ \\
\hline
\end{tabular}

AAL region. The data were then epoched -1.3 to $0 \mathrm{~s}$ relative to stimulus onset (i.e., the aISI period) and separated into conditions (using the same trial balance used for the source maps and peak responses). The alpha power time courses for each AAL region were then averaged over trials and aISI time window within each attention condition and subject. The outcome of this processing was $15 \times 4$ (Experiment 1: $15 \mathrm{AAL}$ regions and 4 attention conditions) or $26 \times 6$ (Experiment 2: 26 AAL regionsincluding AAL regions in the left hemisphere but excluding AAL regions within the somatosensory cortex - and six attention conditions) alpha power values per subject. Data of Experiment 2 were averaged between AAL regions across hemispheres by flipping the attention conditions, resulting in $13 \mathrm{AAL}$ datasets per subject.

Before averaging over subjects, the resulting $4 / 6$ alpha power values for the attention conditions in Experiments 1 and 2 per AAL region were normalized by the alpha power value of the attention condition that showed the maximum power, removing between-subject variance to ensure between condition variance was interrogated. Given the apparent linear and quadratic modulation patterns derived from the peak location analysis, for each AAL region the normalized alpha power averaged over all subjects (i.e., 15/19 data points per condition for Experiments 1 and 2, respectively) were first fit with a linear function. Subsequently, those AAL regions, where no significant linear modulation was observed, were investigated for potential quadratic modulations. This approach was chosen to circumvent the issue that quadratic models; being more complex, will always provide a better goodness of fit than a linear model. Significance of the fits obtained on the real data was determined through Monte Carlo permutation tests (25,000 repetitions). Here, for every AAL region, the real data fits were compared with surrogate distributions of linear and quadratic terms of the respective AAL regions, derived from shuffling data between the different attention conditions for every subject individually and performing new linear and quadratic fits over the $4 / 6$ surrogate attention conditions. The $p$-values obtained were then corrected for multiple comparisons (i.e., AAL regions) using false discovery rate (FDR) correction (Benjamini and Hochberg, 1995; Yekutieli and Benjamini, 1999).

For those regions where a significant quadratic modulation was found, we further interogated whether the quadratic model out-performed a linear model by computing the Akaike Information Criterion (AIC; Akaike, 1974) using the fitlm function implemented in MATLAB. This ruled out the possibility that the significant quadratic modulation was only based on the higher complexity of the model compared with a linear model. The "winner" of these different model types is the one that minimizes the AIC. An ANOVA implemented in the fitlm function tests whether the "winning" model explains the data better than a constant model. The resulting $p$-values were then Bonferroni corrected taking into account the number of AAL regions which showed a significant quadratic modulation.

Control time-frequency analysis. To investigate power lateralization effects due to attentional modulation on a more broad spectrum of frequencies, we conducted a wavelet analysis for frequencies ranging from 1 
A Multi-modal (MM); $N=19$
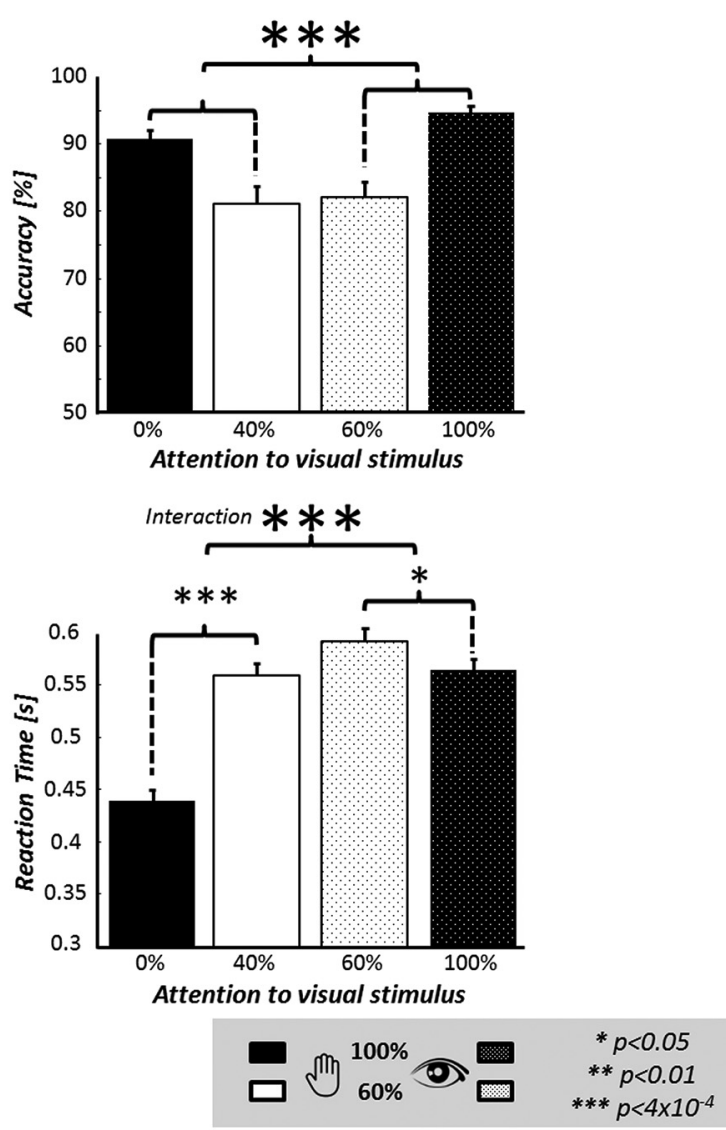

B
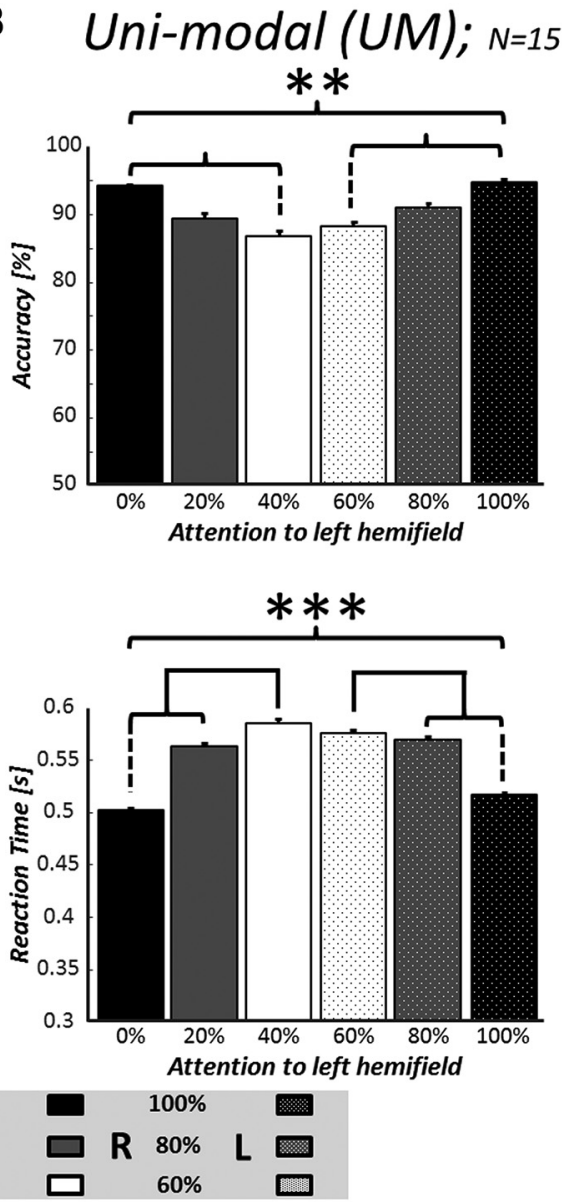

Figure 2. Behavioral measures of accuracy (top) and RT (bottom) across attention conditions. $A$, Behavioral results of the multimodal (visual vs somatosensory) paradigm (top: accuracy achieved in each condition, bottom: RTs). A significant interaction between cue and attended modality in the RT shows that participants' behavior is modulated to a greater extent when attention is directed to the somatosensory modality ( $0 \%$ and $40 \%$ ) than the visual modality (60\% and 100\%). B, Behavioral data for the unimodal (visual) paradigm (top: accuracy achieved; bottom: RTs). All bars denote the mean response over subjects. Error bars indicate the SEM over subjects. Asterisks denote $p$-values from two-way ANOVAs (see caption).

to $48 \mathrm{~Hz}$, using an increasing number of cycles $(2$ cycles at $1 \mathrm{~Hz}$ and 8 cycles at $48 \mathrm{~Hz}$ ) in a time window ranging from $-1.5 \mathrm{~s}$ until $-0.1 \mathrm{~s}$ with respect to stimulus onset. In Experiment 1, this analysis was performed for four neighboring electrodes over right somatosensory areas and four neighboring electrodes over right visual areas (cf. topography plot in Fig. 6). For Experiment 2, four neighboring electrodes over left and four neighboring electrodes over right visual recording sites were chosen (cf. topography plot in Fig. 6). Power lateralization was calculated in the same way as the AMI using Equation 1 (see above). For Experiment 2, right hemisphere electrodes were mirrored to combine with data recorded over left electrodes.

\section{Results}

\section{Behavior}

Experiment 1 (multimodal task)

A two-way repeated-measures ANOVA with main factors of cue ( 100 or $60 \%$ attention) and modality (attention to visual or somatosensory modality) revealed that the accuracy for discrimination of spatial and temporal frequencies was significantly higher in the "attend 100\%" condition than in the "attend 60\%" condition ( $p$-value $=1.3 \times 10^{-7} ; F=34.3$, Fig. $2 A$, top). No significant effect of modality $(p$-value $=0.21 ; F=1.6)$ and no interaction between cue and modality was observed ( $p$-value $=$ $0.4 ; F=0.67$; Fig. $2 A$, top).

When investigating potential differences of the second dependent variable, RTs across attention conditions, we could observe a main effect of cue $\left(p\right.$-value $\left.=1.1 \times 10^{-8} ; F=41.8\right)$. Further- more, a significant interaction between factors cue and modality $\left(p\right.$-value $\left.=1.03 \times 10^{-4} ; F=16.9\right)$ revealed a stronger effect of cue on RTs when subjects attended to the somatosensory stimuli (Fig. 2A, bottom)

\section{Experiment 2 (unimodal task)}

A 2-way repeated-measures ANOVA with main factors of cue $(60 \%, 80 \%$, and $100 \%$ attention) and side of presentation (left or right visual hemifield) revealed as the main effect that the first dependent variable, accuracy for discriminating the orientation of Gabor gratings was significantly higher in the "attend $100 \%$ " than in the "attend 60\%" condition $\left(p\right.$-value $=3.37 \times 10^{-4} ; F=$ 8.8; Fig. $2 B$, top). No significant effect of side of presentation $(p$-value $=0.63 ; F=0.2)$ and no interaction between cue and side of presentation was observed ( $p$-value $=0.64 ; F=0.4$; Fig. $2 B$, top).

Furthermore, RT was significantly shorter when subjects only attended to one side of the visual field ( $100 \mathrm{vs} 0 \%$ attention), than when they divided their attention between hemifields ( 80 vs $20 \%$ and 60 vs $40 \%$ attention; $p$-value $=4.1 \times 10^{-6} ; F=14.4$ ). There was no significant interaction between cue and side of presentation $(p$-value $=0.3 ; F=1.1 ;$ Fig. $2 B$, bottom $)$.

\section{EEG responses}

As there was hypothesized to be more than one alpha power response from different cortical areas, we focus the results on the 
A WHOle BRAin estimate: $100 \% \mathrm{~V}-100 \% \mathrm{~S}$
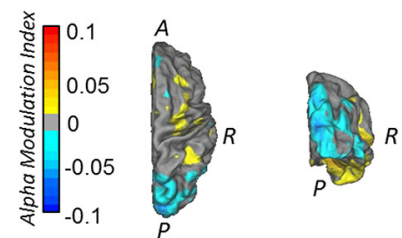

B AAL REGIONS: $100 \%$ V vs. $100 \% \mathrm{~S}$
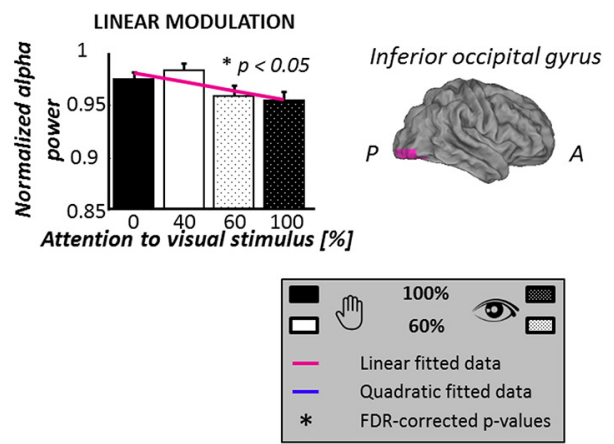

C WHOLE BRAIN ESTIMATE: $100 \% \mathrm{~V} / \mathrm{S}-60 \% \mathrm{~V} / \mathrm{S}$

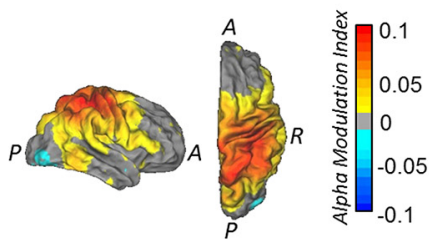

D AAL REGIONS: $100 \% \mathrm{~V} / \mathrm{S}$ Vs. $60 \% \mathrm{~V} / \mathrm{S}$
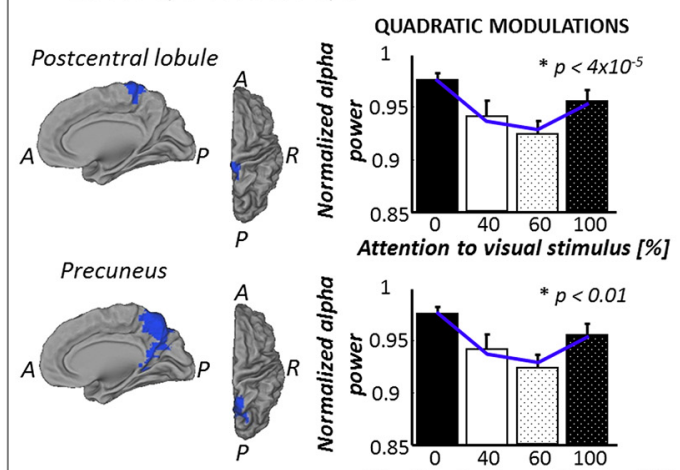

Figure 3. Source analysis results of Experiment 1.A, Source analysis results for Experiment 1 when contrasting the conditions $100 \%$ visual ( $0 \%$ somatosensory) with $100 \%$ somatosensory ( $0 \%$ visual) attention; AMI map of the responses overlaid on the MNI brain (blue denotes regions where alpha power decreased with increasing visual attention). $\boldsymbol{B}$, AAL region where significant linear modulation across conditions was observed. The region identified was the inferior occipital gyrus (marked in pink, $p=0.02$, FDR corrected). The modulation in this region is plotted in the bar graph (average normalized alpha responses across subjects) along with the line of best fit (pink line). C, AMI map obtained when contrasting trials where subjects attended to only one modality (i.e., $100 / 0 \%$ condition) with those where attention was divided (i.e., $60 / 40 \%$ condition) overlaid on the MNI brain (red/yellow denotes regions where alpha power increases when attention is paid to a single modality compared with divided attention). The largest AMI effect to this contrast was in the right parietal area where an increase in alpha power is seen during $100 \% / 0 \%$ attention conditions compared with $60 \% / 40 \%$ conditions. D, AAL regions where significant quadratic modulation across conditions was observed. Both regions identified were in the parietal cortex [postcentral lobule $\{p$-value $=0.003$ (FDR corrected) $\}$ and precuneus $\{p$-value $=0.01$ (FDR corrected) $\}$ ]. Interrogation of the alpha power responses in these regions, shown by the bar graphs ( $\boldsymbol{D}$, right), revealed a "u"-shaped across attention conditions in both regions. Error bars on all bar graphs indicate SEM across subjects for the normalized alpha responses.

source level where spatial localization aids interpretation of the data.

\section{Experiment 1}

To investigate potential differences in alpha power between the attention conditions, we first compared trials where subjects only paid attention to visual stimuli (100\% V; cond 1 in Eq. 1) with trials where they only attended to somatosensory stimuli (100\% $S$; cond 2 in Eq. 1). We computed the alpha modulation index ('AMI'; Eq. 1) on the beam former results, which revealed a negative response in right visual cortex, indicating an alpha power decrease in visual cortex with increasing attention to the visual domain as shown in Figure $3 A$. No alpha power modulation was found in somatosensory areas between these two attention conditions (Fig. 3A). The AAL analyses supported this observation revealing a significant linear modulation of alpha power $(p$ value $=0.02$, FDR corrected; $\left.r^{2}=0.056\right)$ observed in the right inferior occipital gyrus; Figure $3 B$.

To investigate whether differential alpha power modulation was observed in other brain regions in trials where attention was divided between modalities compared with those where attention was focused on one modality only, the AMI between the $100 \%$ (cond 1 in Eq. 1) and 60\% (cond 2 in Eq. 1) attention conditions was computed. This contrast revealed a peak source location in the right parietal cortex, showing higher alpha power in the 100\% than $60 \%$ attention conditions (Fig. 3C, denoted by red color). AAL analysis confirmed this result, showing significant quadratic modulations of alpha power in two superior parietal regions: right postcentral lobule $\left(p\right.$-value $=4 \times 10^{-5}$, FDR corrected; $\left.r^{2}=0.12\right)$ and right precuneus ( $p$-value $=0.01$, FDR corrected; $\left.r^{2}=0.068\right)$. Visual inspection of the alpha power across conditions showed that significantly lower alpha power was induced in these regions when attention was divided between modalities than when subjects paid attention to only one modality (Fig. 3D, left). No significant linear modulations were seen in these regions. To rule out that the significant quadratic modulations over these two AAL regions were purely a result of the higher complexity of quadratic models compared with linear models, we directly compared whether a linear or a quadratic model better explained the data, using the Akaike Information Criterion (AIC; Akaike, 1974). For both regions, the quadratic model minimized the AIC compared with a constant or linear model. Furthermore, in the right postcentral lobule, the quadratic model was significantly better than a constant model (corrected $p=0.022$ ) but failed significance for the right precuneus (corrected $p=0.155$ ).

\section{Experiment 2}

In this second experiment, AMI analysis in source space (Eq. 1) identified a maximum in left and a minimum in right visual cortices when contrasting trials where subjects attended $100 \%$ to the left (cond 1 in Eq. 1) with trials where subjects attended 100\% to the right (cond 2 in Eq. 1) side of the visual field (Fig. 4A). Alpha power at the peak in the left visual cortex increased with increasing attention to the left visual hemifield, whereas the response in the right visual cortex showed a decrease in alpha power. For increasing attention to the right visual hemifield, the 

A WHOLE BRAIN ESTIMATE:
$100 \%$ L- $100 \% R$

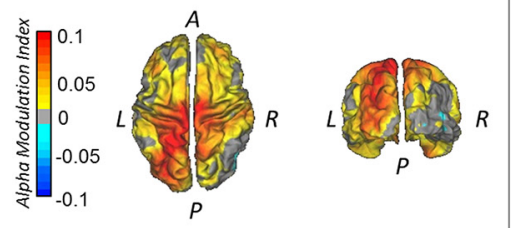

B LINEAR MODULATIONS
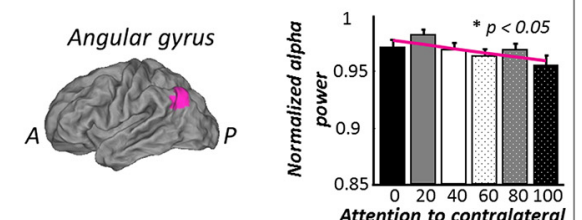

Attention to contralateral hemifield [\%]
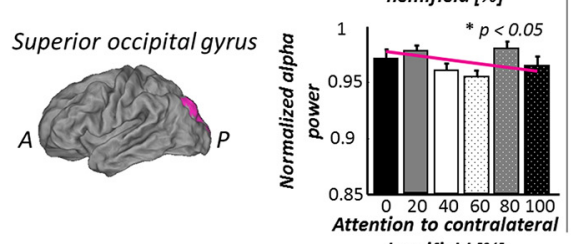

hemifield [\%]

\section{WHOLE BRAin EStimate: $100 \% L-60 \% L$}

$100 \% R-60 \% R$

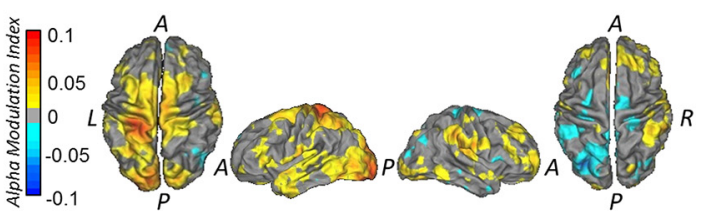

D QUADRATICMODULATION
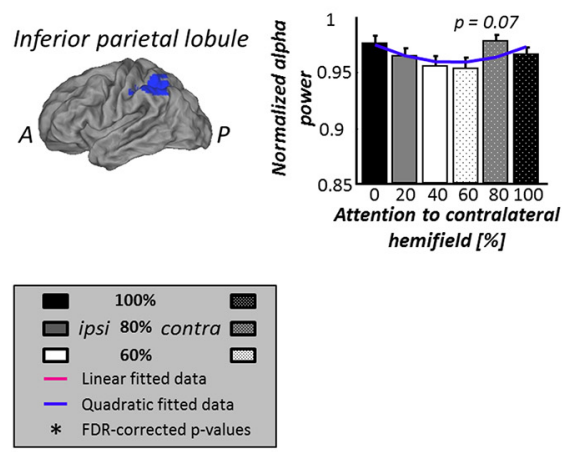
hemifield [\%]

Figure 4. Source analysis results of Experiment 2.A, AMI map when contrasting conditions $100 \%$ attention left versus $100 \%$ attention right (left) revealing an increase (red/yellow color) in alpha power over left visual and parietal areas for the $100 \%$ attention left condition compared with the $100 \%$ attention right condition (the contrast $100 \% \mathrm{R}-100 \% \mathrm{~L}$ would just be the inverse of this AMI map). $\boldsymbol{B}$, Results of the AAL analysis revealing the angular gyrus ( $p=0.03$, FDR corrected) and the superior occipital gyrus ( $p=0.03$, FDR corrected) as the regions with a significant linear modulation of alpha power across the attention conditions (regions shown in pink on the MNI brain). Bar plots show the alpha power over all conditions, again combined for the right and left hemisphere, the line of best fit is shown in light blue. $C$, AMI map when contrasting the attention conditions where participants attended to only one side of the visual field (100\% $\mathrm{L} / \mathrm{R})$ with those when they divided their attention between left and right hemifields ( $60 \% \mathrm{~L} / \mathrm{R})$ overlaid on an MNI brain. The left images show the responses to attention modulation to the left visual field, and the brain maps on the right show the same modulations with attention to the right visual field. The AMI maps show increase over ipsilateral parietal and visual areas to that side where attention is paid when attention is directed fully to that spatial location ( $100 \%$ condition) compared with divided between locations ( $60 \%$ condition). $\boldsymbol{D}$, Results of the AAL analysis with a trend of a quadratic modulation over the inferior parietal lobule ( $p=0.07$, FDR corrected). The bar plot shows the alpha power over all conditions, again combined for the right and left hemisphere, the line of best fit is shown in dark blue. Error bars on all bar graphs indicate SEM across subjects for the normalized alpha responses.

opposite was observed. Therefore, a decrease in alpha power could be observed over visual areas contralateral to the focus of attention whereas an increase in alpha power was present over visual areas of the hemisphere ipsilateral to attention. These responses were combined by inverting the responses across conditions measured from right hemisphere and then averaging with those measured from left hemisphere. The results of this analysis are shown in the bar plot in Figure $4 A$, and suggest a linear modulation of alpha power by attention. The fitting analysis in AAL regions confirmed this observation, showing that with increasing attention, alpha power decreased linearly over visual areas of the hemisphere contralateral to the focus of attention: significant linear fits were found in the angular gyri $(p=0.03$, FDR corrected; $\left.r^{2}=0.052\right)$ and superior occipital gyri ( $p=0.03$, FDR corrected; $\left.r^{2}=0.047\right)$, as shown in Figure $4 B$.

The AMI maps comparing the conditions $100 \%$ attention (cond 1 in Eq. 1) and 60\% attention (cond 2 in Eq. 1) revealed maxima in the parietal cortex in the hemisphere ipsilateral to where visual attention was directed (Fig. 4C, AMI maps), indicating higher alpha power in the $100 \%$ attention conditions than the $60 \%$ attention conditions. Interrogation of these responses over all conditions showed a quadratic ("u"-shaped) alpha power modulation pattern, as shown in the bar plot in Figure 4C. Further interrogation using the AAL analysis showed that a trend ( $p=0.07$, FDR-corrected; $\left.r^{2}=0.039\right)$ for a quadratic fit was observed over the parietal region inferior parietal lobule (IPL). Visual inspection of the alpha power across conditions for this
AAL region, revealed that the quadratic fit was a " $u$ " shape (Fig. $4 D$ ), as seen in the peak analysis (Fig. 4C) and similar to that seen for the multimodal paradigm shown in Figure 3, $C$ and $D$.

\section{Discussion}

Numerous EEG/MEG studies showed that posterior alpha power is modulated by attention. However, it is unclear whether these alpha power modulations reflect one or several attentional mechanisms. Here we show using EEG source analysis (LCMV beam former) that in unimodal and multimodal attention tasks, alpha power is differently modulated by attention in occipital and parietal areas (Fig. 5).

We found two alpha sources, visual and parietal, which can be separated spatially and experimentally. These sources are both modulated by attention, but play different functional roles depending on behavioral demands. The visual alpha source showed linear power decreases with increasing attention to visual stimuli or a given location, thus indicating the location of attention, i.e., the visual Spotlight of Attention (Posner et al., 1980; Crick, 1984; Eriksen and Yeh, 1985). In contrast, the parietal alpha source was quadratically modulated by attention showing lower alpha power when attention was divided, between modalities or spatial locations, rather than focused on either. Thus we suggest the parietal alpha source likely indicates attentional effort. Regions showing significant linear (pink) and quadratic (blue) alpha power modulations observed in Experiments 1 and 2 are summarized in Figure 5. 

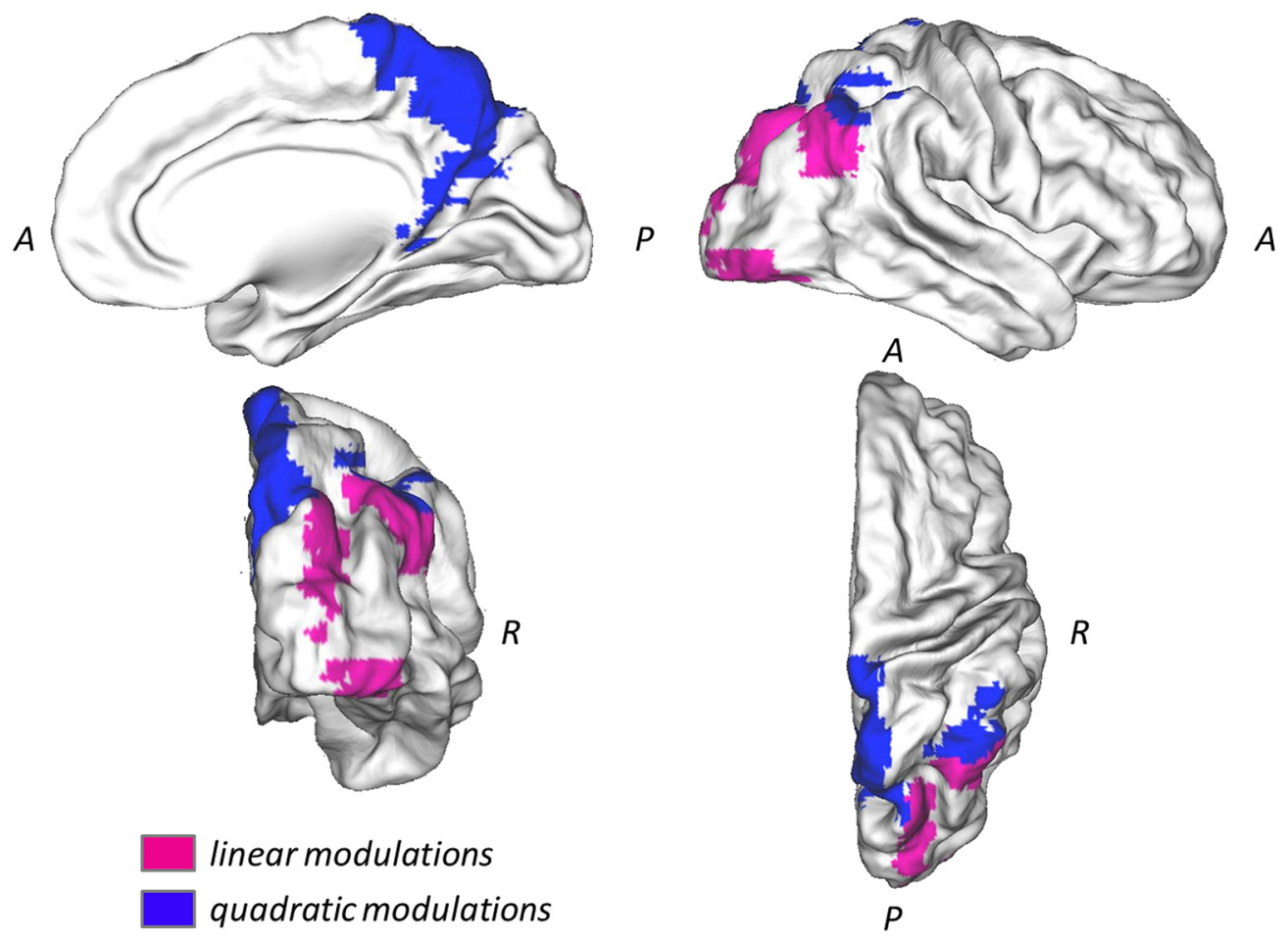

Figure 5. Summarizing alpha power modulation effects with attention. To simplify, all effects are shown on the right hemisphere. AAL regions show significant linear (pink) and significant/trend quadratic (dark blue) modulations of al pha power. A, Anterior; P, posterior; R, right.

On the behavioral level, we have replicated previous findings (Gould et al., 2011) and show a robust effect of attention, modulating significantly accuracy and RTs in both experiments where higher accuracy and lower RTs are present when attention is focused on one modality (Exp. 1) or spatial location (Exp. 2).

Surprisingly, we did not find an alpha power lateralization effect over somatosensory regions, when comparing attention to visual and somatosensory targets in Experiment 1 . We thus investigated the data using a broad frequency spectrum $(1-48 \mathrm{~Hz})$ and found that the power lateralization between visual and somatosensory recording sites seems to depend more on beta $(\sim 16-17 \mathrm{~Hz})$ than alpha oscillations. Although the data of the purely visual task of Experiment 2 show a prominent peak in the alpha frequency band ( $\sim 10 \mathrm{~Hz}$; cf. Fig. 6, right), Experiment 1 shows a more broad effect, with a peak frequency in the beta band (cf. Fig. 6, left). We think that this could be the reason that we did not find any linear attention modulation effects on alpha power over somatosensory areas. Although this finding is interesting, the focus of this study was the role of alpha oscillations in different types of attention. Further analyses of this beta band effect are therefore subject to future reinvestigation of this dataset.

\section{Functional significance}

The two tasks used in this study show similar clustering of alpha activity in visual and parietal areas (Fig. 5). Although quadratic modulations over parietal areas that were observed in the purely visual task (Exp. 2) only showed a trend toward significance, this still suggests that the two alpha sources are a general phenomenon of attention rather than specific to the experimental task. If our assumption is true, the imprecise region of interest that has previously been reported as parietooccipital is actually composed of two distinct brain sources that act in different ways. We hypothesize that the sensory-specific source reflects "the visual spotlight of attention" and is controlled by top-down processes coming from a parietal alpha source, which in turn is modulated by attentional effort toward the task. Participants reported that the purely visual task of Experiment 2 was easier to accomplish than the multimodal task in Experiment 1. This discrepancy could contribute to the weaker effect of quadratic modulations over parietal areas in Experiment 2.

Previous fMRI studies showed that both visual and parietal regions show an increased BOLD response in the hemisphere contralateral to the direction of visual spatial attention (Sylvester et al., 2007; Bressler et al., 2008; Lauritzen et al., 2009), reflecting increased cortical excitability. Using Granger causality, Bressler et al. (2008) further showed that the FEF and IPS, both part of the dorsal attention network, were responsible for driving neural activity in early visual areas by top-down control. Other studies obtained similar results (Ruff et al., 2008; Marshall et al., 2015; Popov et al., 2017). Because EEG alpha activity and the BOLD signal are widely reported to be negatively correlated (Goldman et al., 2002; Laufs et al., 2006; Scheeringa et al., 2011), an increase in BOLD signal over contralateral visual and parietal areas in a visual spatial attention task could be related to a decrease in alpha activity over the same regions, which would agree with the findings of this study. Capotosto et al. (2009) hypothesized that topdown control from frontal and parietal areas mediates the occipital alpha rhythm and therewith the level of inhibition. Using rTMS to inhibit the previously identified regions FEF and IPS (Bressler et al., 2008), Capotosto et al. (2009) also showed increased RTs and decreased accuracy for target detection. Furthermore, they demonstrated that this inhibition abolished the prestimulus alpha-desynchronization, which can be typically observed over parietal and occipital electrodes contralateral to attention and concluded that this disruption in top-down control of the visual alpha rhythm led to a decrease in visual identification (Capotosto et al., 2009). However, they were not able to identify what neuronal rhythms from IPS and FEF were causing this top- 

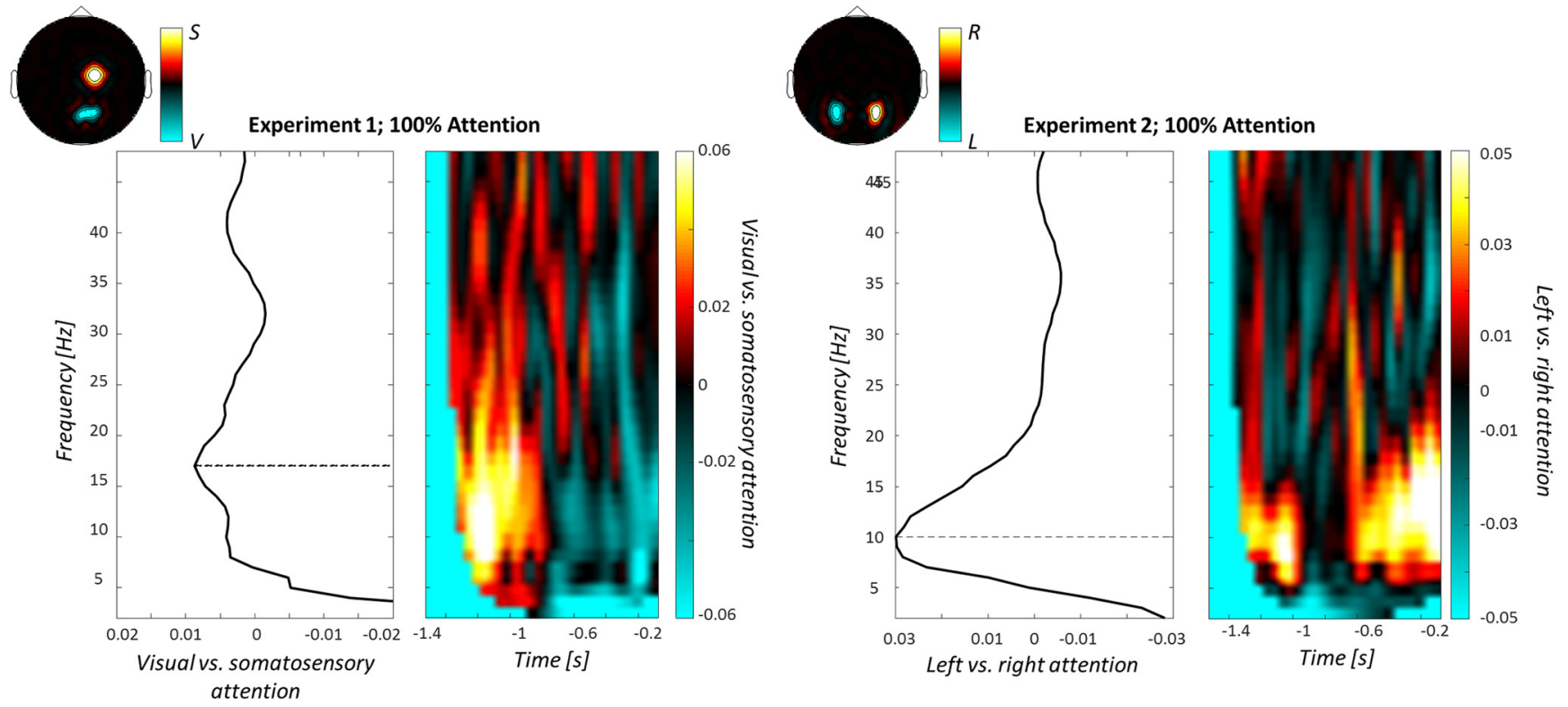

Figure 6. Power lateralization effect in multimodal and unimodal attention paradigm. Broad-frequency analysis of power lateralization effect revealed a peak in the beta frequency band $(\sim 16-17 \mathrm{~Hz})$ for Experiment 1 (left) and a prominent peak in the alpha band $(\sim 10 \mathrm{~Hz})$ for Experiment 2 (right).Power lateralization was computed over four neighboring somatosensory and visual electrodes [Experiment 1; highlighted in topography plot on the left as S (somatosensory) and V (visual)], as well as over four neighboring left and right visual electrodes [Experiment 2; highlighted in topography plot on the right as $\mathrm{R}$ (right) and $\mathrm{L}$ (left)].

down control of the occipitoparietal alpha rhythm from their experiment.

Previous studies support the idea for spatially distinct visual and parietal alpha sources mediating attention and visual perception. Van Dijk et al. (2008) showed that low prestimulus parietal alpha power was advantageous for visual discrimination (van Dijk et al., 2008). They concluded that this parietal alpha source regulates alpha power in low-level visual areas via top-down control. With our results we could reinterpret their findings and conclude that the parietal alpha power modulation rather reflects the attentional state than the level of inhibition of the occipital cortex. Thus, low parietal alpha power would indicate a state where the subject is engaged in the task, leading to the recruitment of top-down attention and an increase in performance in discriminating grating orientations or temporal and spatial frequencies. Another example is a recently published EEG study (Gulbinaite et al., 2017) on the triple-flash illusion, where a third visual flash is perceived upon presentation of only two stimuli. The illusion comes about when presenting the second stimulus after a specific interval; the authors could show that this interval and the illusory percept correlated with the individual alpha frequency at parietal but not occipital sources. Consistent with these findings, a recent intracranial EEG study shed more light onto these distinct alpha sources and their directionality, supporting the view of a top-down control of occipital alpha by parietal areas (Halgren et al., 2017). The authors recorded resting state data on epilepsy patients and found evidence for alpha generators in the parietal cortex. They further showed that alpha acts like a traveling wave, propagating in space from parietal to occipital brain regions (Halgren et al., 2017). Albeit the important evidence for the existence of two spatially distinct alpha sources, none of the above presented studies could experimentally dissociate them into occipital and parietal sources.

Crucially we extend these previous studies by showing that there are two distinct alpha sources which are modulated differentially by attention in two different sets of experiments and are thus likely to have different functional roles. These data adds to a growing body of evidence that there are multiple alpha sources present during a cognitive task with distinct roles (Nunez et al., 2001). Alpha oscillations have gained much interest in neuroscientific research and their image has changed from reflecting a passive idling state (Pfurtscheller et al., 1996) to actively regulating inhibition in the service of cognition (Klimesch et al., 2007; Palva and Palva, 2007; Jensen and Mazaheri, 2010; Mathewson et al., 2011). Given the ubiquity of alpha oscillations in the human brain, it makes sense to assume that the role played by alpha is a very general one like gating neural activity. If this assumption is true, then we should be able to dissociate different alpha oscillations in a complex cognitive task that recruits a number of cortical assemblies controlled by alpha. Our data represents such evidence where we spatially and experimentally dissociate an occipital/ventral parietal from a more superior parietal alpha source in two experiments requiring a complex interaction between topdown and bottom-up attention processes. Previous studies focused on the role of alpha in gating low level sensory information (Jensen et al., 2012). We add to this literature by showing that alpha not only indicates the locus of attention, but also the recruitment of higher order areas, which arguably control the shift of attention to lower-order, primary sensory locations/modalities. Our results open up the avenue for future non-invasive human EEG studies to investigate how alpha oscillations in these two regions coordinate their activity to implement attentional shifts, which so far has mostly been addressed by invasive animal recordings (von Stein et al., 2000; Buffalo et al., 2011; van Kerkoerle et al., 2014).

EEG source localization relies on whether the assumptions of its algorithm are met by the data. Our EEG results are corroborated by a separate, high resolution fMRI study conducted by our group at ultra-high field ( $7 \mathrm{~T}$ ) on a subsample of the same subjects (7/10 participants also performed Experiment 1), using the same multimodal task as in Experiment 1. This study (Aquino et al., 2019) also reveals two fMRI sources modulated by attention: 
(1) quadratic BOLD-response modulations over parietal areas when contrasting attentional effort (100\% vs 60\%) and (2) linear modulations over visual areas when contrasting the location of attention (100\% vs 100\%). Due to the superior spatial resolution of fMRI, these results strongly suggest that the two alpha band sources measured with EEG are indeed distinct sources that need to be considered separately.

\section{Conclusions}

We show that two spatially distinct alpha sources execute different roles in unimodal and multimodal attention: (1) a parietal source modulated by attentional effort showed significantly lower alpha power when subjects divided their attention, which potentially exerts top-down control on alpha oscillations over lower-level visual areas; and (2) a visual alpha source that reflects the current spotlight of visual attention showing a significant linear power decrease with increasing attention to visual stimuli, possibly driven by top-down control from parietal alpha sources. Given that such a top-down control has been shown to exist (Ruff et al., 2008; Bressler et al., 2008; Capotosto et al., 2009), we hypothesize a similar top-down regulation from parietal toward visual areas; however, further exploration is needed to confirm this hypothesis.

\section{References}

Akaike H (1974) A new look at the statistical model identification. EEE Trans Autom Control 19:716-723.

Anderson KL, Ding M (2011) Attentional modulation of the somatosensory mu rhythm. Neuroscience 180:165-180.

Aquino KM, Sokoliuk R, Pakenham DO, Sanchez-Panchuelo RM, Hanslmayr S, Mayhew SD, Mullinger KJ, Francis ST (2019) Addressing challenges of high spatial resolution UHF fMRI for group analysis of higher-order cognitive tasks: An intersensory task directing attention between visual and somatosensory domains. Hum Brain Mapp 40:12981316.

Bauer M, Kennett S, Driver J (2012) Attentional selection of location and modality in vision and touch modulates low-frequency activity in associated sensory cortices. J Neurophysiol 107:2342-2351.

Bell AJ, Sejnowski TJ (1995) An information-maximization approach to blind separation and blind deconvolution. Neural Comput 7:1129-1159.

Benjamini Y, Hochberg Y (1995) Controlling the false discovery rate: a practical and powerful approach to multiple testing. J R Stat Soc Ser B (Methodological) 57:289-300.

Brainard DH (1997) The psychophysics toolbox. Spat Vis 10:433-436.

Bressler SL, Tang W, Sylvester CM, Shulman GL, Corbetta M (2008) Topdown control of human visual cortex by frontal and parietal cortex in anticipatory visual spatial attention. J Neurosci 28:10056-10061.

Brookes MJ, Tewarie PK, Hunt BAE, Robson SE, Gascoyne LE, Liddle EB, Liddle PF, Morris PG (2016) A multi-layer network approach to MEG connectivity analysis. Neuroimage 132:425-438.

Buffalo EA, Fries P, Landman R, Buschman TJ, Desimone R (2011) Laminar differences in gamma and alpha coherence in the ventral stream. Proc Natl Acad Sci U S A 108:11262-11267.

Capotosto P, Babiloni C, Romani GL, Corbetta M (2009) Frontoparietal cortex controls spatial attention through modulation of anticipatory alpha rhythms. J Neurosci 29:5863-5872.

Corbetta M, Shulman GL (2002) Control of goal-directed and stimulusdriven attention in the brain. Nat Rev Neurosci 3:201-215.

Crick F (1984) Function of the thalamic reticular complex: the searchlight hypothesis. Proc Natl Acad Sci U S A 81:4586-4590.

Eriksen CW, Yeh YY (1985) Allocation of attention in the visual field. J Exp Psychol Hum Percept Perform 11:583-597.

Foxe JJ, Snyder AC (2011) The role of alpha-band brain oscillations as a sensory suppression mechanism during selective attention. Front Psychol 2:154.

Foxe JJ, Simpson GV, Ahlfors SP (1998) Cognitive neuroscience. Neuroreport 9:3929-3933.

Goldman RI, Stern JM, Engel J Jr, Cohen MS (2002) Simultaneous EEG and fMRI of the alpha rhythm. Neuroreport 13:2487-2492.
Gomez-Ramirez M, Kelly SP, Molholm S, Sehatpour P, Schwartz TH, Foxe JJ (2011) Oscillatory sensory selection mechanisms during intersensory attention to rhythmic auditory and visual inputs: a human electrocorticographic investigation. J Neurosci 31:18556-18567.

Gould IC, Rushworth MF, Nobre AC (2011) Indexing the graded allocation of visuospatial attention using anticipatory alpha oscillations. J Neurophysiol 105:1318-1326.

Gulbinaite R, İlhan B, VanRullen R (2017) The triple-flash illusion reveals a driving role of alpha-band reverberations in visual perception. J Neurosci 37:7219-7230.

Haegens S, Händel BF, Jensen O (2011) Top-down controlled alpha band activity in somatosensory areas determines behavioral performance in a discrimination task. J Neurosci 31:5197-5204.

Haegens S, Luther L, Jensen O (2012) Somatosensory anticipatory alpha activity increases to suppress distracting input. J Cogn Neurosci 24:677-685.

Halgren M, Devinsky O, Doyle WK, Bastuji H, Rey M, Mak-McCully R, Chauvel P, Ulbert I, Fabo D, Wittner L, Heit G, Eskandar E, Mandell A, Cash SS (2017) The generation and propagation of the human alpha rhythm. Available from: https://www.biorxiv.org/content/10.1101/202564v1.

Jensen O, Mazaheri A (2010) Shaping functional architecture by oscillatory alpha activity: gating by inhibition. Front Hum Neurosci 4:186.

Jensen O, Bonnefond M, VanRullen R (2012) An oscillatory mechanism for prioritizing salient unattended stimuli. Trends Cogn Sci 16:200-206.

Kelly SP, Lalor EC, Reilly RB, Foxe JJ (2006) Increases in alpha oscillatory power reflect an active retinotopic mechanism for distracter suppression during sustained visuospatial attention. J Neurophysiol 95:3844-3851.

Klimesch W, Sauseng P, Hanslmayr S (2007) EEG alpha oscillations: the inhibition-timing hypothesis. Brain Res Rev 53:63-88.

Laufs H, Holt JL, Elfont R, Krams M, Paul JS, Krakow K, Kleinschmidt A (2006) Where the BOLD signal goes when alpha EEG leaves. Neuroimage 31:1408-1418.

Lauritzen TZ, D’Esposito M, Heeger DJ, Silver M a, Wills H, HHW Jr (2009) Top-down flow of visual spatial attention signals from parietal to occipital cortex. J Vis 9:18.1-18.14.

Marshall TR, O’Shea J, Jensen O, Bergmann TO (2015) Frontal eye fields control attentional modulation of alpha and gamma oscillations in contralateral occipitoparietal cortex. J Neurosci 35:1638-1647.

Mathewson KE, Lleras A, Beck DM, Fabiani M, Ro T, Gratton G (2011) Pulsed out of awareness: EEG alpha oscillations represent a pulsedinhibition of ongoing cortical processing. Front Psychol 2:99.

Nunez PL, Wingeier BM, Silberstein RB (2001) Spatial-temporal structures of human alpha rhythms: theory, microcurrent sources, multiscale measurements, and global binding of local networks. Hum Brain Mapp 13: $125-164$.

Oostenveld R, Fries P, Maris E, Schoffelen JM (2011) FieldTrip: Open source software for advanced analysis of MEG, EEG, and invasive electrophysiological data. Comput Intell Neurosci 2011:156869.

Palva S, Palva JM (2007) New vistas for $\alpha$-frequency band oscillations. Trends Neurosci 30:150-158.

Pfurtscheller G, Stancák A Jr, Neuper C (1996) Event-related synchronization (ERS) in the alpha band-an electrophysiological correlate of cortical idling: a review. Int J Psychophysiol 24:39-46.

Popov T, Kastner S, Jensen O (2017) FEF-controlled alpha delay activity precedes stimulus-induced gamma-band activity in visual cortex. J Neurosci 37:4117-4127.

Posner MI, Snyder CR, Davidson BJ (1980) Attention and the detection of signals. J Exp Psychol Gen 109:160.

Robinson S, Vrba J (1998) Functional neuroimaging by synthetic aperture magnetometry. In:Recent Advances in Biomagnetism (Yoshimoto T, Kotani M, Kuriki S, Karibe H, Nakasato N eds), pp 302-305. Sendai, Japan: Tohoku University Press.

Ruff CC, Bestmann S, Blankenburg F, Bjoertomt O, Josephs O, Weiskopf N, Deichmann R, Driver J (2008) Distinct causal influences of parietal versus frontal areas on human visual cortex: evidence from concurrent TMSfMRI. Cereb Cortex 18:817-827.

Scheeringa R, Fries P, Petersson KM, Oostenveld R, Grothe I, Norris DG, Hagoort P, Bastiaansen MC (2011) Neuronal dynamics underlying high- and low-frequency EEG oscillations contribute independently to the human BOLD signal. Neuron 69:572-583.

Sylvester CM, Shulman GL, Jack AI, Corbetta M (2007) Asymmetry of an- 
ticipatory activity in visual cortex predicts the locus of attention and perception. J Neurosci 27:14424-14433.

Tadel F, Baillet S, Mosher JC, Pantazis D, Leahy RM (2011) Brainstorm: A user-friendly application for MEG/EEG analysis. Comput Intell Neurosci 2011:879716.

Thut G, Nietzel A, Brandt SA, Pascual-Leone A (2006) -band electroencephalographic activity over occipital cortex indexes visuospatial attention bias and predicts visual target detection. J Neurosci 26:9494-9502.

Tzourio-Mazoyer N, Landeau B, Papathanassiou D, Crivello F, Etard O, Delcroix N, Mazoyer B, Joliot M (2002) Automated anatomical labeling of activations in SPM using a macroscopic anatomical parcellation of the MNI MRI single-subject brain. Neuroimage 15:273-289.

van Dijk H, Schoffelen JM, Oostenveld R, Jensen O (2008) Prestimulus oscillatory activity in the alpha band predicts visual discrimination ability. J Neurosci 28:1816-1823.

Van Drongelen W, Yuchtman M, Van Veen BD, Van Huffelen AC (1996) A spatial filtering technique to detect and localize multiple sources in the brain. Brain Topography 9:39-49.

van Ede F, de Lange F, Jensen O, Maris E (2011) Orienting attention to an upcoming tactile event involves a spatially and temporally specific modulation of sensorimotor alpha- and beta-band oscillations. J Neurosci 31:2016-2024. van Kerkoerle T, Self MW, Dagnino B, Gariel-Mathis MA, Poort J, van der Togt C, Roelfsema PR (2014) Alpha and gamma oscillations characterize feedback and feedforward processing in monkey visual cortex. Proc Natl Acad Sci U S A 111:14332-14341.

Van Veen BD, van Drongelen W, Yuchtman M, Suzuki A (1997) Localization of brain electrical activity via linearly constrained minimum variance spatial filtering. IEEE Trans Biomed Eng 44:867-880.

von Stein A, Chiang C, König P (2000) Top-down processing mediated by interareal synchronization. Proc Natl Acad Sci U S A 97:14748-14753.

Waldhauser GT, Braun V, Hanslmayr S (2016) Episodic memory retrieval functionally relies on very rapid reactivation of sensory information. J Neurosci 36:251-260.

Worden MS, Foxe JJ, Wang N, Simpson GV (2000) Anticipatory biasing of visuospatial attention indexed by retinotopically specific-band electroencephalography increases over occipital cortex. J Neurosci 20:RC63.

Yekutieli D, Benjamini Y (1999) Resampling-based false discovery rate controlling multiple test procedures for correlated test statistics. J Stat Plan Inference 82:171-196.

Zumer JM, Scheeringa R, Schoffelen JM, Norris DG, Jensen O (2014) Occipital alpha activity during stimulus processing gates the information flow to object-selective cortex. PLoS Biol 12:e1001965. 